\title{
Photoacoustic Tomography: High-resolution Imaging of Optical Contrast In Vivo at New Depths
}

\author{
Lihong V. Wang, Fellow, IEEE
}

\begin{abstract}
Existing high-resolution 3D optical imaging cannot provide penetration into biological tissues beyond one transport mean free path $(\sim 1 \mathrm{~mm}$ in the skin). Photoacoustic tomography, which combines strong optical contrast and high ultrasonic resolution in a single modality, has broken through this fundamental depth limitation.
\end{abstract}

Key words: Photoacoustic tomography, photoacoustic imaging, photoacoustic computed tomography, photoacoustic microscopy, optical imaging

$\mathrm{U}$ nlike ionizing $\mathrm{x}$-ray radiation, non-ionizing electromagnetic waves, such as optical and radio waves, pose no health hazard yet can provide new contrast to medical imaging. Unfortunately, electromagnetic waves in the non-ionizing spectral region do not penetrate biological tissue in straight paths.

Despite the high optical contrast, pure optical imaging is currently limited by either the spatial resolution or the imaging depth. High-resolution optical tomography, such as confocal microscopy and two-photon microscopy as well as optical coherence tomography, is limited to a depth of approximately one transport mean free path $(\sim 1 \mathrm{~mm})$. Lowresolution diffuse optical tomography (DOT), by contrast, can provide near-infrared functional imaging of the breast and brain. Light around $700 \mathrm{~nm}$ in the near-infrared spectral region can penetrate multiple centimeters of biological tissue. However, recovering imaging information with high spatial resolution from diffuse photons remains a challenge. As a rule of thumb, the spatial resolution of DOT is about $10 \%$ of the sample thickness.

High-resolution photoacoustic tomography has been developed as a hybrid imaging modality for functional and molecular imaging. Ultrasonic imaging provides good image resolution but poor contrast in early-stage tumors; in addition, it has strong speckle artifacts. Ultrasound-mediated photoacoustic imaging combines optical and ultrasonic waves synergistically to overcome the aforementioned problems with pure optical or ultrasonic imaging. Photoacoustic tomography uses low-energy laser pulses to induce ultrasonic waves, referred to as photoacoustic waves, in biological tissue. Detection of the photoacoustic waves

L. V. Wang is Gene K. Beare Distinguished Professor, Optical Imaging Laboratory, Department of Biomedical Engineering, Washington University

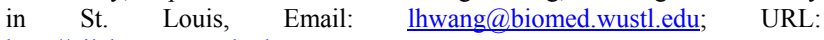
http://oilab.seas.wustl.edu. yields speckle-free images with strong optical contrast at high ultrasonic resolution in relatively large volumes of biological tissue.

Photoacoustic tomography refers to cross-sectional or 3D imaging based on the photoacoustic effect. Although the photoacoustic effect as a phenomenon was first reported on by Alexander Graham Bell in 1880, photoacoustic tomography as an imaging technology emerged only recently with the advent of ultrasonic transducers, computers, and lasers. In this talk, I will introduce the two major forms of photoacoustic tomography: photoacoustic computed tomography and photoacoustic microscopy. The latter will be discussed in detail.

Photoacoustic microscopy, which combines high ultrasonic resolution and strong optical contrast in a single modality, has broken through the fundamental penetration limit at the optical transport mean free path (Table 1). While photoacoustic imaging is sensitive to optical absorption, other modalities are sensitive to optical scattering or fluorescence. The various modalities complement each other in both penetration and contrast and therefore will co-exist.

Table 1. Comparison of high-resolution optical imaging modalities.

\begin{tabular}{|l|l|l|}
\hline Modality & Penetration & Contrast \\
\hline Confocal microscopy & $\sim 0.5 \mathrm{~mm}$ & $\begin{array}{l}\text { Scattering, } \\
\text { fluorescence }\end{array}$ \\
\hline $\begin{array}{l}\text { Two-photon } \\
\text { microscopy }\end{array}$ & $\sim 0.5 \mathrm{~mm}$ & Fluorescence \\
\hline $\begin{array}{l}\text { Optical coherence } \\
\text { tomography }\end{array}$ & $\sim 1 \mathrm{~mm}$ & $\begin{array}{l}\text { Scattering, } \\
\text { polarization }\end{array}$ \\
\hline $\begin{array}{l}\text { Confocal } \\
\text { photoacoustic } \\
\text { microscopy }\end{array}$ & $\begin{array}{l}\sim 3-30 \mathrm{~mm}, \\
\text { scalable }\end{array}$ & Absorption \\
\hline
\end{tabular}

The spatial resolution and the maximum imaging depth can be scaled with the ultrasonic bandwidth within the reach of photons. For example, when operating at $50 \mathrm{MHz}$ ultrasonic detection frequency, photoacoustic microscopy can image up to $3 \mathrm{~mm}$ into scattering biological tissue with 15 micrometer axial resolution. When operating at $5 \mathrm{MHz}$, photoacoustic microscopy can image $\sim 10$ times as deep but with $\sim 10$ times axial resolution. 